\title{
A Mind Selected by Needs: Explaining Logical Animals by Evolution
}

\author{
Fabian Seitz ${ }^{1}$ (D)
}

Received: 29 April 2019 / Accepted: 7 January 2020 / Published online: 20 January 2020

(C) The Author(s) 2020

\begin{abstract}
Explaining humans as rational creatures - capable of deductive reasoning - remains challenging for evolutionary naturalism. Schechter (Philosophical Perspectives, $24(1) 437-464,2011,2013)$ proposes to link the evolution of this kind of reasoning with the ability to plan. His proposal, however, does neither include any elaborated theory on how logical abilities came into being within the hominin lineage nor is it sufficiently supported by empirical evidence. I present such a theory in broad outline and substantiate it with archeological findings. It is argued that the cognitive makeup of any animal is constituted by being embedded in a certain way of life. Changing ways of life thus foster appearances of new cognitive abilities. Finally, a new way of life of coordinated group behavior emerged within the hominins: anticipatory group planning involved in activities like making sophisticated spears for hunting. This gave rise to human logical cognition. It turned hominins into domain-general reasoner and adherents of intersubjective norms for reasoning. However, as I argue, it did not—and most likely could not-give rise to reason by deductive logic. More likely, deductive reasoning entered our world only a few thousand years ago: exclusively as a cultural artifact.
\end{abstract}

Keywords Cognitive evolution · Reasoning · Niche construction · Cognitive archeology Deductive logic $\cdot$ Cultural evolution

\section{Introduction}

Naturalizing human reasonable agency persists a central challenge for the current philosophy of mind. Examples of daily activities quickly turn into examples fraught with problems. Consider Maryam. She is contemplating whether to minor in anthropology or mathematics. Her major subject is philosophy, so she wants a reasonable

Fabian Seitz

F.Seitz@em.uni-frankfurt.de

1 Institut für Philosophie, Johann Wolfgang Goethe-Universität, Norbert-Wollheim-Platz 1, D-60629 Frankfurt am Main, Germany 
complement for her affinity. She thinks about it thoroughly and eventually decides to go for anthropology. Mary does not know it, but she is a miracle. From the standpoint of the metaphysics of logic, that is.

Why is that? First, she is a sentient living organism. She has an evolutionary history and, correspondingly, a distinct internal organization and specific needs. Like all animals, she possesses abilities for object-directed cognition and purposive behavior.

Second, Mary did two things: she believed one thing and then another and finally combined these beliefs with one of her desires to form an intention on what to do. Reasoning did both: on the one side, to figure out what to believe and on the other, to deliberate and to reflect on what to do, henceforth being able to justify her doings in retrospect. Thereby, she can demarcate good lines of thoughts from flaws. She has some feeling for what counts as good justification. If she is right that philosophy should have an empirical basis and anthropology is such a base, then studying anthropology is a sound choice. It feels true that it follows. However, why believe in such truths at all?

Some rely on a "logical given" to explain this phenomenon. Logical laws are construed as constituting "an objective realm" independent of our existence, nonetheless guiding our thinking as norms: timeless and spaceless entities, building the deepest layer of reality over and above the contingent empirical world. What a human agent therefore needs is a capacity to recognize these never changing "logical laws."

However, if humans are nothing but natural items, it should be possible to explain their characteristic features by the natural components they are made of. Naturalism is obliged to comprehend everything that exists as finite, spatiotemporally embedded things. Human logical reasoning and everything attached to it would have to be naturalized in the same way. Yet this is not a wieldable option. It requires explaining how non-trans-spatiotemporal beings can recognize trans-spatiotemporal principles.

These are all entangled issues, but they ultimately boil down to the following: how is logical cognition possible as an evolved trait? (And henceforth the cognition of abstract logical objects and the following of abstract, apparently trans-spatiotemporally valid logical norms in one's episodes of reasoning.) The proposal to be elaborated here is that our logical mind evolved due to advanced planning abilities.

In Section 3, I develop a niche construction model able to explain how logical cognition had to occur within the hominin lineage to enable their way of life. In Section 4, empirical data to support this model is presented. ${ }^{1}$ By this, I argue that advanced planning abilities necessitated logical cognition: What can be identified is selection for domain-generality and adherence to intersubjective norms. I return to the question of logic then. Following my model, it is unlikely that a need for using the standard set of deductive rules of logic (as known from logic courses) emerged during hominization. The outlook is that deductive reasoning and abstract logical laws more likely entered our world only a few thousand years ago: exclusively as cultural artifacts. Hence, I end by sketching the idea that logical laws are not trans-spatiotemporal, for we created these items ourselves. Consequently, logic's normative status depends on

\footnotetext{
${ }^{1}$ This kind of philosophizing will depend heavily on exploiting data, but it is still philosophy, not science. Its aims are philosophical, too: to foster debates on the nature of rationality and abstract objects, on their epistemology, and so on. Moreover, giving an account of how logic might have evolved is part of a core project of recent analytic philosophy of mind: to explain human minds as part of nature.
} 
context and is instrumental at most. To start, I outline Joshua Schechter's ideas on the evolution of deductive reasoning.

\section{Evolving Answers}

\subsection{Planning and Logic}

Schechter $(2011,2013)$ has offered an evolutionary "in principle" explanation for our faith in logical truths. It is a typical one for cutting-edge naturalists about logic, so I use it as my starting point. ${ }^{2}$ He wonders about our more-or-less granted "reliability" about logical truths, for by and large "our logical beliefs match the logical facts" (2013: p. 215). Schechter construes these facts as the logical truths of classical deductive logic: objective, abstract, employing the feature of necessary truth preservation (2011: pp. 441-443; see also 2013: p. 216). Explaining this reliability remains a challenge, however, for Schechter accepts Benacerraf-style worries of how to align oneself with abstract (logical) objects: after all, no causal-perceptual contact with them is possible (2013: pp. 216-219). We do not know "how we could be reliable about objective facts that were not learned via some kind of perception" (2013: 216). Thus, Schechter proposes to rely on a more "basic competence": our ability to reason deductively. This proposal changes the topic from the input side of cognition to central internal practice. Instead of asking how we could recognize these abstract entities, he construes our reliable beliefs about them as "a byproduct of the general ability of our cognitive mechanism for deductive reasoning" (2013: pp. 237-238). This mechanism "conferred a heritable [...] reproductive advantage upon our ancestors" (2013: p. 216).

But what is this kind of reasoning for? How did it become part of the hominin gene pool? Any idea of adaptation implies that there are always concrete answers to concrete environmental conditions, whereas deductive reasoning is the ability to reason domaingenerally about whatsoever, hence with no particular environmental "enabling conditions" that would select for this kind of reasoning. As Schechter puts it, "[e]volutionary accounts $[. .$.$] seem unable to explain how it is that we employ deductive rules that are$ reliable for arbitrary subject matters" (2013: p. 230; emphasis in the original). Consequently, we need to "explain [...] why it is that we employ reliable deductive rules" (2013: p. 231).

Here, Schechter follows the general idea that these rules evolved to foster long-term planning abilities (2013: p. 235ff.; see footnote 2 for earlier formulations of this idea by others). Such planning relies on the incorporation of high loads of information from both, first, different cognitive sources and, second, gathered at different spots and at different times. Furthermore, as a social animal engaged in group activities, there could also be the need to coordinate one's action with others (2013: p. 231). ${ }^{3}$ According to Schechter, reasoning with deductive rules would be helpful here.

He suggests something along the following lines: deductive rules are a byproduct of the rules that evolution originally selected for in hominins. Our ancestors might have

\footnotetext{
${ }^{2}$ Compare, for entirely different accounts about logic, but similar evolutionary ideas, Cooper (2001) and Stenning and van Lambalgen (2008).

${ }^{3}$ I agree with all three observations. See Section 3 for how I handle these cases.
} 
been under pressure to work only a "limited range of propositions" (2013: p. 232). However, they had also been under pressure to find a "computationally efficient" (ibid.) way to work an ever-growing amount of information. According to Schechter, deductive reasoning is a suitable candidate mechanism to satisfy the latter condition. ${ }^{4}$ Furthermore, for Schechter having (logical) concepts grounds the ability to reason (logically). Now, having abstract logical concepts like and, or and similar ones is a better way to work information than with special purpose concepts. (Think of wisentand-willow, tiger-or-safety, and so on.) Again, it would be computationally inefficient to "dispense with general-purpose logical concepts in favor of a large number of special purpose concepts." (2013: p. 232). Once these concepts are part of the hominin's mind, hominins turn into deductive reasoners.

Let me abstract. Although this is not Schechter's wording, his account could be described as a turnaround strategy: instead of starting to believe in logical truths, we originally evolved to follow deductive rules of reasoning. Only then we start to believe in the former. Consequently, all epistemological problems vanish, for these truths do only "embody" rules we already use. A somewhat magical, but established, co-variance between our evolved cognitive rules and these abstract logical objects occurred. In this way, he aspires to avoid the problems such as the ontological and normative nature of logic. ${ }^{5}$ So far, I agree with him - in broad outline that is.

\subsection{Why Possible Is Not Enough}

Problems arise with the details. Most urgently, his account does not give an empirically supported explanation of how extensive planning could have evolved alongside our reasoning abilities. To be fair, Schechter does not aim for such details. He only seeks for a philosophical possibility, not an empirical explanation (2013: p. 230). Due to this, however, his whole line of thought rests on the conceivability of one premise: that planning with high information load will possibly select for deductive reasoning.

In what follows, I will not discuss whether conceivability entails (evolutionary?) possibility - let us take this for granted here. Also, I will not criticize Schechter's way of arguing. I esteem his method and know too well that everyone can look it up in the standard playbook of analytic philosophy. Also, I completely agree with referring to philosophy mainly as "in the business of promoting possibilities" (Hutto and Myin 2013: xi). Yet "promoting possibilities" without empirical background is not enough in the specific case of establishing evolutionary naturalism about some phenomenon.

Granted Schechter's strategy works out, anti-evolutionary skepticism about logic remains, and rather strong, for he can only argue for evolvability of deductive reasoning at some worlds: the subset of worlds where circumstances make his premise true there - whatever these circumstances might be in detail. Yet he has not demonstrated that our world belongs to this set. He has also not argued that deductive reasoning necessarily had to evolve for hominins. However, there is no contradiction in holding that $x$ is possible, but actually not the case. Any anti-evolutionist can retreat to such a position, and all the more, the more unlikely $x$ is. They could argue that, while

\footnotetext{
${ }^{4}$ A line of thought already elaborated by Sober (1981)

${ }^{5}$ See Section 5 for further characterization of these problems.
} 
deductive reasoning might be possible to evolve, it is either unlikely to happen or even could not happen in our lineage for contextual reasons.

And indeed, adding some context from our actual world and a little bit of evolutionary theory downsizes the likelihood of Schechter's premise considerably. To start with, there is a strong consideration against the evolution of deductive reasoning. Even if a trait is within the range of possible variations, there must be a need for it to be selected for. Schechter apprehends correctly that local scenarios are improbable to come up with situations in which the ability to reason deductively would be needed (Schechter 2013: p. 230). Hence, he must ask what deductive reasoning would allow for in a specific case, contrary to non-deductive forms. Fixation on the feature of necessary truth preservation will not help to settle this issue, but to focus more pragmatically on making counterexamples will. To translate, Schechter needs a scenario which fosters its inhabitants to reason towards a plan with absolutely no counterexamples possible to any intermediate transitions of their episodes of reasoning. He further would have to argue that our ancestral environment was such that these counterexample-free patterns were frequently more beneficial for survival than others. Only then deductive reasoning would be a candidate mechanism to be selected for.

Yet even advanced anticipatory planning does not require patterns of reasoning which are safe against all possible counterexamples. To suggest that this is the general core of our ancestor's argumentative practices seems to be somewhat overkill. On that level, certainty is hard to achieve in real-world scenarios. Moreover, it is not needed to guarantee a successful threshold for executing plans, too. Real interactions in real environments always depend on finite sets of real encounters. But these encounters are the only resources available to evolved beings. This is a case against the idea that planning selects for deductive reasoning.

Furthermore, Schechter's scenario is not a very strong case in itself. In the following, my critique mirrors Richardson's (2008) arguments that many adaptationist explanations for psychological mechanisms are under-supported by the available evidence (Richardson 2008, chapter 2; see Walter 2009 for a reconstruction of his arguments).

The core of Schechter's a priori consideration is that a design feature of modern logic would be a good solution to the problem of "high information load": general concepts and rules are computationally more efficient than specific ones. Hence, if there is a need to work vast amounts of information, having general concepts and rules turns out to be beneficial. This might be true. But evolution does not work that way.

First, selection can only work on existing variations. One needs proof that the preferred option is among the possible variations. Second, one cannot deduce from the statement mentioned above that evolution automatically is going to select for this trait: even if beneficial, if there are other options available, one needs proof that the other options are not a better pay-off. Finally, every trait at a given scenario builds on a preceding trait of a previous scenario, and often, this pre-version itself had to be adaptive in the past. There must be a precursor as a "platform" for a trait to evolve. 6

\footnotetext{
${ }^{6}$ For example, extant hunter-gatherers have tremendous knowledge of their surroundings (Saslis-Lagoudakis et al. 2014). Early hunter-gatherers might not have been so different in this regard. If so, building upon already existing memory abilities to store vast amounts of both concepts and specific reasoning rules might have been a more economical solution than inventing a new cognitive mechanism.
} 
Yet to consider which candidates are actually possible, whether there were better pay-offs available or from which "platform" a trait could have evolved requires information about particular circumstances at a given time. And one needs empirical evidence to describe these circumstances.

Here his account falters. To demonstrate possible-that- $x$ proofs not-necessarily-not$x$, but it says nothing about whether our ancestors lived in an $x$-world. True, it suffices to avoid a knock-down argument on behalf of the anti-evolutionist ("deductive reasoning could never evolve, anywhere"). Yet this is only a partial defense of evolutionary naturalism and not a great leap forward in making a case for it. For, in general, evolution more likely does not select for deductive reasoning rather than doing it. All things equal and given no evidence to the contrary, anti-evolutionists can claim that although humans are evolved beings, their ability to make use of deductive logic more likely does not derive from our evolutionary inheritance. Ergo, it might have a nonevolutionary source.

Anti-evolutionists can balance the debate to their favor as long as evolutionists do not have a strong argument that Schechter's premise had to be true for our ancestor's world — or more realistically: at least more plausible than alternatives.

\subsection{From Possible to Plausible}

Schechter's attempt to establish evolutionary naturalism shifted the dispute-willingly or not - from arguments about possibility towards arguments about plausibility. As just seen, the route from a philosophical "possible" to an evolutionary "plausible" is more intricate than expected: without empirical support, a possible scenario still leaves too much room for maneuvers to dismiss, if not the genuine possibility, at least the plausibility of such a scenario. To adequately describe such scenarios requires to integrate diverse knowledge from different disciplines: anthropological, archeological, biological, geological, paleontological, and so on. Schechter's possible just-so scenario takes none of this into account. This stance might be all right for a philosophical thought experiment - for tevolution on twin earth, say. Yet it falls short of proving what's at issue: to demonstrate whether our actual logical abilities could have evolved in our concrete world. Since the argumentative weight rests on dealing with an aspect of our actual world, one does not require a "universalist" explanation how the occurrence of the phenomena could be explained "in principle" somewhere, but how it could have happened at our place - in the specific context of hominization.

Therefore, evolutionary naturalists will have to argue that evolution forced our ancestors to reason deductively. In a nutshell, we need an empirically plausible scenario to make a case for evolutionary naturalism about logic. Furthermore, such an empirically supported account must be composed out of two elements: first, a reasoned statement why animals within a specific context would need this specific trait; second, a description of how hominins could end up - historically - in such a context at all.

My conjecture is that such an account is achievable. For despite the critique given in the previous section, I remain guardedly optimistic about just-so stories (pace Richardson). True, given the fragmentary character of available evidence, this might not always work out. Nevertheless, constructing stories is part of any explanation of adaptations, and also constructed stories have their virtues: they order the available evidence in the most coherent way, thereby being constrained multiple times by the 
very same evidence, and they build a base to form additional hypotheses to either further confirm or dismiss any preliminary story (Currie and Sterelny 2017). Every attempt to reconstruct a part of history will incorporate uncertainties to some extent: the point is to make an explanation as coherent as possible with different streams of evidence to avoid the common mistake of letting the (desired) outcome determine how the explanation unfolds (Smith 2016).

Also, Schechter offers excellent ideas to build upon. He has changed the topic from the evolution of how we came to cognize abstract objects to the evolution of an essential cognitive skill: reasoning. Furthermore, he has located the origin of this ability in the context of the ability to coordinate behavior. In what follows, I fill the lacuna of his account with these starting points. As a result, we gain a new evolutionary account which explains why and how hominins turned into domain-general reasoner and adherents of intersubjective norms for reasoning. As argued above, this did not give rise to reason by deductive logic, however. In Section 5, I conjecture that deductive reasoning might be a more recent accomplishment. Now, to get this account started, let me sketch how cognition evolves according to my proposal. ${ }^{7}$

\section{What Is a Mind for?}

\subsection{Needs}

To begin with, I assume the following as a reasonable scheme. An animal has a need, crucial for survival, hence the goal to meet this need. Reaching that goal involves producing a new state of the world. It does so by changing the arrangement of things (or some things themselves) within its reach. Thus, having needs fosters the need to manipulate things. Furthermore, manipulating things attaches to needs not only in this straightforward way. Things can even create new needs after they have become part of the animal's inventory. "Basic needs" then unfold into branching out sub-needs. All of this sets the stage for the dynamics of cognition.

To explain what an animal's mind is for in this setting, I follow Godfrey-Smith's (1996) basic idea: "the immediate role of cognition is to control behavior" (GodfreySmith 2002: p. 5f.). It is a means to produce behavioral flexibility, in order "to deal with environmental complexity" (Godfrey-Smith 2002: p. 5f.; see also 1996: p. 13). Depending on its needs and habitat, an animal must be capable of a certain level of behavioral flexibility. This forms the complexity of a mind, for animals have to carry out specific behaviors. This requires whatever particular cognitive abilities the animal needs to conduct this behavior. All this in response to a given environment it has to cope with.

How does behavior then form the cognitive skill of reasoning? First, I propose the following: if cognition is all about controlling behavior, then the activity of reasoning is there to control behavior, too. ${ }^{8}$ Second, behaviors unfold in line with a

\footnotetext{
${ }^{7}$ Here a caveat is in order: what follows in Sections 3 and 4 is a sketch of a much bigger project to come. In line with this, my aim in this paper is only to give the first glimpse of this project and to provide a "proof of concept" for it.

${ }^{8}$ As stated here, it is only an assumption to get started. It will have to vindicate itself by enabling a reasonable evolutionary trajectory in the long run.
} 
certain complexity. They reach from a state of awareness of a need to a goal state, thereby tackling obstacles on the way. The behavioral sequence consists of different steps here, which must be executed in the right order. Therefore, there is a need for coordination: to coordinate the different subparts of behavior in a way as to achieve the primary goal. Otherwise, successful behavior would not be possible. Now, this need to properly coordinate the parts of a sequence fosters the need to integrate all information required, as it were, from a "vantage point." One must always have the ability to work any information necessary for a task. It grounds the need to integrate all the information required: information across many domains, depending on the single parts and subtasks involved.

In other words: both kind and amount of information to be combined are set by this requirement for coordination. This requirement, in turn, is relative to the behavior that has to be coordinated. Eventually, cognitive skills are selected for combining all this in such a way as to put a behavior into practice with chances of success. Following my proposal, reasoning prepares and accompanies this practice. If so, the function of (proto-)reasoning would be to work up information in such a way that it enables the right orchestration of behaviors. Reasoning, then, is this activity of coordinating behavioral parts and information: to construct behavioral sequences in mind and to incorporate information before and during execution.

What finally determines the cognitive work, however, are the things used to meet one's needs, for they determine the required behavior sequences. I elaborate how in the next section.

\subsection{The Cognitive Burden of Things}

There is yet another aspect of Godfrey-Smith's framework. Achieving goals depends on how an animal has to interact with its environments. Thus, there is a relation between the kind of environment to the particular behavior. Complex environments tend to demand complex behaviors. The latter tend to demand complex cognitive skills. Here, Godfrey-Smith's notion of "environmental complexity" enters. There is more than one way to spell out this notion (Godfrey-Smith 2017); yet to characterize the complexities involved here, I am going to rely on Hodder's (2012) idea of entanglement. (Note that nothing in the overall argument depends on this choice: the account presented below would also work with other ways to characterize environmental complexities.)

Entanglement is a set of relationships. Agents relate to things and things to agents, and even things itself often relate to each other. Entanglements create specific dependencies thereby. First, agents have to rely on things to adapt to their environment. These things enable them to meet their needs. Second, they have to care for these items, for artifacts "cannot reproduce on their own" (Hodder 2014: p. 30). Hence one has "to look after them, repair them, replace them, manage them" (2014: p. 30). Control of these artifacts and their components is crucial (cf. 2014: p. 33). This implies an important aspect. Depending on things to meet one's needs made one even more dependent. It demands to cope with everything necessary to get these things in the first place. Here, the notion of entanglement can be used as a tool for fixing analytical units. Agents use artifacts, but one should not regard artifacts as fixed entities, instead, as a set of dependencies, which have to be considered from an agent's perspective. 
It might sound trivial, but this agent-relative notion of thing-skill-entanglement makes a difference in explaining cognitive evolution, for artifacts foster human thought in many ways. ${ }^{9}$ Creating and sustaining them is one possibility. It requires cognitive resources of its own. Starting to use artifacts creates subordinated needs. One has to transform raw materials. Forming the material into its shape is required. Knowing where to get raw material from, and include this into one's daily routines, is required, too. One also needs to know how to maintain an artifact.

Artifacts thus demand certain cognitive abilities due to these connections. An artifact's entanglements demand a particular manipulation of the environment to handle them. It requires skills in decision-making and resource management. Entanglements thus imply cognitive activities such as planning. In the abstract, an agent has to manipulate things for meeting one's primary needs. The entanglements of these things create the complexity an agent has to cope with. The latter characterizes cognitive demand then. Thus, the entanglements of things drive the complexity of cognition.

This order can be reversed for analysis. To some extent, one can read off from an artifact the entanglements an agent (from his standpoint) has to handle. For this, knowledge of the particular ecology must be integrated, too. Then, one can also read off the required handling of knowledge for control and resource management. The latter then allows for reasoned speculation about the cognitive abilities used. Thus, one can analyze from an artifact which knowledge had to be handled and which general rules of thought had to be followed to get this very artifact. ${ }^{10}$

This also holds true for our ancestors and us. During hominin history, we happened to become depended on particular things to fulfill our needs. Our entanglements specified the minimal requirements of cognitive abilities to handle them. All things we once were entangled with thus drove our cognitive evolution. I turn now to this step in the overall loop.

\subsection{Niche Construction: First Steps}

Niche construction is a systematic change, which organisms bring about within their environments (Day et al. 2003). These changes can alter selective pressures for themselves and their offspring. Modified niches change the selective regimes for the next generation. This can become an important factor in the evolutionary dynamics of a species. A prime example is a selection for lactose tolerance in those Homo sapiens groups (and only those), which engaged in the practice of dairying (Boivin 2008: p. 200f.). For my purpose, two aspects stand out. First, niche construction plays a role within the cognitive capacities of its inhabitants, for niche construction and cognitive adaptions interact. Second, niche construction can result in evolutionary feedback, for "organisms drive environmental change and organism-modified environments subsequently select organisms" (Laland and Brown 2006: p. 96). The latter again alter their environments for their descendants.

Niche construction can alter cognitive development directly. Take, for example, the transformation thesis of Sutton (2010) and Menary (2007, 2015: p. 8f.). A

\footnotetext{
${ }^{9}$ There are more accounts worth mentioning, of course, but for reasons of space, I concentrate on my proposal.

${ }^{10}$ This is akin to the chaîne opératoire-approach in archeology (Leroi-Gourhan 1965).
} 
transformation of cognitive abilities can occur due to change in the cultural niches. Menary's cognitive integration theory, for instance, maintains, "along with niche constructionists (Laland et al. 2000), ... a phylogeny of hominid cognition in terms [of] their active embodiment in a socially constructed niche" (2015: p. 3). Constructing new niches hence leads to new cognitive abilities. ${ }^{11}$ This change is based on the idea that the mind extends beyond the brain. Hominin minds couple with all kinds of artifacts to perform cognitive tasks. If the artifacts change, so does cognition. But Menary (and Sutton) employ here a specific class of things: cognitive artifacts (Hutchins 1999). Examples of such artifacts are writing and number systems. These are instances of a more general phenomenon: humans invent both material artifacts and practices, which in turn alter their cognitive abilities. ${ }^{12}$

One can give this a more historical bend. Osvath and Gärdenfors (2004) (henceforth O\&G) also employ niche construction. But they analyze the cognitive evolution of early hominins. Furthermore, unlike extended mind theorists, they concentrate on noncognitive artifacts. These can have transformative effects on cognitive evolution, too. For, according to O\&G, a changing hominin niche 2.5 MYA caused coevolution of transport and planning. Transportation of raw materials and tools expanded at that time. Hence, behavior sequences stretched more and more. Finally, according to O\&G, anticipatory planning became mandatory, for hominins planned these behavior sequences. Yet to handle these ever longer transports, they had to plan in anticipatory mode at a certain level.

However, I will focus on another aspect than the mere length of behavior sequences. Recall that cognition can change through time due to a change of material things surrounding it, for changing artifacts within a niche is also changing their entanglements. For the agents involved, this changes which items of an environment they have to track and cope with. Thus, it selects for cognitive skills to handle this.

With this framework provided now, I explain why the reasoning abilities of our ancestors became both domain-general and bound to intersubjective norms. ${ }^{13}$ In any event, cultural change is the motor of cognitive evolution in the hominins.

\section{Hominin Cultures: Extended Manufacture and Maintenance}

Time to flesh out the account with empirical evidence. Plausible scenarios based on the archeological record on hominin evolution will be sketched. ${ }^{14}$ I link evidence on extensive planning with the rise of domain-general cognition in Section 4.1. Likewise, in Section 4.2, evidence of cooperative activities like group planning is linked with a rise of adherence to intersubjective norms in hominin reasoning activities.

\footnotetext{
${ }^{11}$ Therefore, "we should be searching for archaic precursors to modern cognitive capacities" (Menary 2015: p. $6)$.

${ }^{12}$ This is, of course, only a highly abbreviated sketch of 4E cognition. Due to lack of space, I cannot elaborate on it any further (for a recent overview see Gallagher 2017, ch.2).

${ }^{13} \mathrm{My}$ proposal is of course similar to other recent niche constructionist proposals on hominin cognitive evolution (Boivin 2008; Henrich 2016; Heyes 2012; Laland 2017; Sterelny 2012).

${ }^{14}$ Leaving the task of constructing an evolutionary story out of these building blocks to another paper. See Fn 20 on p.(last page).
} 


\subsection{Temporal and Spatial Extent}

There is a series of events during hominization, which caused hominins to plan with more and more expanding entanglements. The early hominins were very mobile apes from early on in their history: they needed to travel extensively to meet their needs. They were also capable of reasoning, for these hominins had to plan at least an essential subset of their daily activities (Osvath and Gärdenfors 2004; Jeffares 2010a). Early Australopithecines (3.5 million years ago [MYA]) are associated with long-distance transport of food and material (Jeffares 2010b: 164). Later on, from 1.8 to 1.6 MYA, entanglements increased during the Oldowan niche-probably established by Homo rudolfensis or late Australopithecines (Semaw et al. 1997). This niche is associated with the transport of goods like raw material, tools, and food up to $15 \mathrm{~km}$ as well as the emergence of so-called "accumulation spots." So goods were not only transported, hominins gathered them at certain places in the landscape (Bunn 1994; Hay 1976; Potts 1984; Ohel 1984). The exact function of these sites is disputed, but it is clear that meat and tools were transported to these spots from perhaps kilometers away (Plummer 2004).

The control of fire was a further crucial event during hominization. Controlled fireuse allows first of all to change one's dietary regimes: meat and plants, especially storage organs like tubers, become more easily digestible or digestible at all (Wrangham 2010; Wrangham and Conklin-Brittain 2003). This diet, in turn, makes for smaller guts, thus bigger brains, to which a considerable change of social organization is attached (Wrangham 2010: p. 193). Campfires may have fostered the upcoming of human communicative and innovation skills, as well as a stronger reliance on traditions in general (Suddendorf 2013: p. 251). Hence, it allows for an expansion of both the amount and diversity of artifacts. The material record on controlled fire use is scrappy and ambiguous. Its presumably latest possible occurrence 700,000 years ago is linked to the emergence of late Homo Erectus (Goren-Inbar et al. 2004)—while some argue for an entirely early beginning 1-1.6 MYA (Clark and Harris 1985).

Then, with the succeeding Homo heidelbergensis, there is one astonishing artifact recorded, which exemplifies well the entanglement that gave rise to domain-general cognition: the hunting spears of Schöningen, approximately 400,000 years old (Thieme 1997). With this artifact, entanglements increased, compared with earlier stone tool production. Heidelbergensians had "to cut down young trees" (Coolidge and Wynn 2012: p. 50). Then, they "removed the side-branches, and shaved off the bark and outer layers of wood" (ibid.). Finally, "they then shaved the spears so that they had a center of gravity about one-third of the way from one end, a weighting that resembles that of modern javelins" (ibid.). These single tasks required a set of different stone tools. Also, these tools, in turn, had to be produced in advance.

This production sequence indicates their cognitive sophistication, for they had to fragmentize the overall task into timely separated parts. Otherwise, they could not have handled the entanglements involved. Archeologist Miriam Haidle (2009) argues that the manufacture of the Schöningen spears lasted a day or two. Likewise, in heidelbergensian stone tool production: "[P] reparing raw material and manufacturing tools became separate tasks" (Jeffares 2010a: 167). This selects for the ability to plan anticipatorily. It enabled heidelbergensis to create small action units in mind and then combine them in a modular way (Haidle 2009). 
Such anticipatory planning selects for reasoning abilities, which eventually will apply to all domains of thinking. For, in the long run, it necessitates to reason domain-generally to achieve one's goals with chances of success. Coordinating one's plans over larger spatial and temporal scales increased informational burdens. Local knowledge about the surrounding ecology increased as home ranges extended. It raised both relevant events to be considered and costs of failure. To coordinate these tasks thus required combining more and more diverse, actual local knowledge. Moreover, in uncertain cases, hominins had to argue for or defeat this information.

Hence, to justify information in planning and decision-making changed its character. It involved an increasing ability to account for previously unconnected bits of information. The need to justify information by crossing domains accelerated. Consequently, the risk of combining non-uniform information in ill-fated ways accelerated. Justifying thus became more depending on rules of reasoning that minimized that risk.

As an adaption to this need, these new rules had to be more general to cover all various domains used. For this, these rules had to allow hominins to abstract more and more from the actual content of information. For this, in turn, they needed to employ more general patterns within their reasoning abilities.

Eventually, anticipatory planning makes every single bit of information potentially relevant to each other - even if all information will never be required at once. For at any time one must decide, there will be changing combinations of information to be considered. These bits of information will always be taken from one's overall web of acquirements, but with no foresight which combination could come next. Hence, one must be able to consider all the information accessible to oneself (virtually, that is).

So any hominin niche, which involves the planning activities mentioned above, displays the need to reason domain-generally. This is not the full story, however.

\subsection{Cooperation}

The niche of the hominins required not only temporally extended planning of behavior sequences. It also required socially extended, cooperative planning. Somewhere between 2 and 0.5 MYA, there has been a fundamental shift in hominin lifeway (Sterelny 2007, 2012: pp. 10-18 and pp. 73-77; see also Foley and Gamble 2009). In the long run, the evolution of the hominins brought about a cooperative hunter (Bunn 2007; Bunn 1994; Bunn and Pickering 2010b; Bunn and Pickering 2010a). Those early hominins became dependent on food resources that could only be attained by cooperative activities. According to Sterelny, "meat consumption becomes a key part of hominin life, probably via increasingly aggressive scavenging as a precursor to actual hunting" (2012: p. 77). For this reason, their lifeways depended on a specific foraging style. One that was (and, still is) "information rich, cooperative, and tool-assisted, and it targeted high-value resources" (2012: p. 77). This was neither a solitary nor a smallgroup activity. For these early hominins, economic activity depended on collective action (Sterelny 2012: p. 77). Eventually, with the arrival of the heidelbergensis, a hominin species cooperated to bring down even big game (Villa and Lenoir 2009).

So, probably already before Homo heidelbergensis, Erectines had to cope with a considerable number of situations in coordinated, planned action. More to the point, as home range increases, single individuals had to cope with their parts of coordinated group behavior much more on their own. If so, this created a collective action 
problem of a particular kind. The problem of coordinating one's actions in the face of coordinated group behavior exacerbates when confronted with extended home ranges: spatial distance between single individuals will be too big to coordinate one's actions by signaling to each other; meanwhile, timing requires to execute specific behaviors in specific time frames. Thus, some parts of the overall plan have to be executed in parallel by different agents at the same time. Fire seems to be an artifact priming such scenarios.

Consequently, making decisions on the fly is not just a problem of information, but one of how this information should be worked, too, for all reasoning in place is obliged to be "compatible" among different agents. It must be guaranteed that information would be reworked nearly the same way by every agent. Otherwise, this could lead to unfitting results: the same information would lead to different outcomes in different agents. This sometimes happens-but it cannot be the general rule if any cooperative activity shall ever have any chance of success. Likewise, if agents have to change something on their part of the plan, they must be sure that it will still fit with the rest of the other parts. In principle, these cases are extensions of the need to coordinate: extended from the level of the subject towards the intersubjective level of the group. To establish this requirement, a shared normative set of rules for reasoning is required: a standard - as the only obliged way - on how to work all information needed. Expanded cooperative activities thus select for an adherence to publicly shared norms for reasoning.

To conclude, cooperative anticipatory planning selects for reasoning abilities, which can apply to all domains of thinking, and reasoners urged to follow public norms for thought. With this result, let me return to the issue of deductive logic.

\section{Outlook}

Given the evolutionary explanation of hominin reasoning just outlined, what about positions like Schechter's, which claim that there has been selection for deductive reasoning? As mentioned earlier, such positions mainly suffer from a lack of empirical evidence. Imagining "it would be most useful" is not an evolutionary argument. (Perhaps the situation is different with our tancestors - but, again, this is no help in explaining the phenomenon at issue.) So, what real arrangement of things would foster behavior sequences, which could only be planned by deductive reasoning rules? Which kind of entanglement could make necessary truth preservation a prerequisite? As argued in Section 2, there is no theoretical reason to think such a prerequisite was necessarily required during hominin evolution. So far, there also seems to be no empirical evidence for such an artifact in the niches of the Middle Pleistocene hunters. Hence, there is neither evidence for deductive rules as a universal "model" how the human mind works when engaged in reasoning activities nor much reason to believe in deductive logic as a yardstick for reasoning in general.

Nevertheless, we have deductive logic. Why is that? I would propose the following as a probable explanation. If my account is on the right track, the cognitive prerequisites for deductive logic indeed evolved during hominization. To wit, it is being able to reason domain-generally and being inclined to follow public norms in reasoning. However, as also argued above, such norms for reasoning get always established by 
local circumstances - based on needs but established as cultural artifacts. ${ }^{15}$ If true, the norms for deductive reasoning had to be established in a particular niche due to particular demands.

Following one historical exposition, the deductive method appeared late in human history, ${ }^{16}$ first invented, probably, by members of the Athenian elite 2500 years before present. It arose as a specific argumentative practice within debates: as dialogues with the element of persuading one another (Dutilh Novaes 2015: pp. 595-597, 2012; Netz 1999). Here, this method is advantageous. Granted both participants of a dialogue agree on a shared set of premises, any conclusion drawn by deductive steps from this set should be regarded as entirely compelling. Given a logically valid form, no counterexample can be given which would show that premises could be accepted, but the conclusion would remain open to being denied. Because no countermove is possible, the opponent must accept the conclusion or should revise one of his premises otherwise.

From this perspective, deductive reasoning is like reading, writing, or calculating a socially learned practice. Deductive reasoning is neither an evolved biological "constant" nor a "universal" of Homo sapiens' mind. It is a cognitive ability to be inoculated by a certain practice and only open to those members of a population who has been brought to a specific learning environment. Hence, deductive logic must be inherited by a tradition, and only those who have learned it will be able to reason by deductive rules. ${ }^{17}$ In this sense, it is like any other piece of mathematical notation. We are not "hard-wired" to use analytical algebra, but once this cultural artifact is there and part of our niche, we can put it to use for all kinds of things. The same goes for deductive logic, as I propose here.

Yet the cognitive prerequisites which allowed to invent this cultural artifact evolved long before. Planning indeed made up the ground to allow for something like deductive reasoning to emerge. Most likely, there has been an urgent need for public argumentation ever since we turned into a cooperative species (Mercier and Sperber 2011; see also Tomasello 2014: pp. 142-143). Likewise, domain-general reasoning became compulsory for us since we turned into animals capable of anticipatory planning. Both aspects combined lay the groundwork for the invention of (deductive) logic as a cultural artifact many hundreds of thousand years later. But deductive reasoning itself is not an answer to any of the pressures which gave rise to human logical cognition (in the sense of the ability to reason both domain-generally and guided by public norms). Only the combination of logical cognition with the Athenian niche 2500 years before present did. Hence, deductive logic does not describe an accompanying part of our mind. However, it can become part of our mind, as 4E-theorists have suggested (see Section 3.3). Since deductive logic has an established and stable cultural practice, our mind can use it for further tasks. Once an artifact, deductive logic could be coupled with the mind and in this more twisted sense could become part of it — at least to those who get educated in this practice.

\footnotetext{
${ }^{15}$ It is even possible that the origin of those norms perishes, while they are still handed down from one generation to the other. Compare for a similar case with rules on food processing (Henrich 2016).

${ }^{16}$ My historical sketch here follows the exposition of Dutilh Novaes $(2015 ; 2012)$, herself relying strongly on Lloyd (1996) and Netz (1999).

${ }^{17}$ It is not ruled out that some deductive steps have always been part of the argumentative practices from heidelbergensis onwards. modus ponens and disjunctive syllogism cross one's mind.
} 
Could Schechter (and like-minded collaborators) agree with such a cultural evolutionary account? If so, they would have to change their conception of logic's nature. To repeat, for Schechter, logic consists of unchanging abstract objects, and a reasoner has to adapt to them, albeit indirectly on his account (2011: pp. 441-443; see also 2013: p. 216). Based on my account, logic is the by-product of channeling information in cooperative activities in such a way as to establish successful interaction with their environments. As such, logic is neither uniform nor steady. The ongoing development of ways to cope with one's environments, and hence the need to find new ways to argue, fosters logic always anew.

With these considerations established, let me end with some speculative sketches:

Abstracta. Grasping abstract logical objects as cultural artifacts devours challenges about their ontology. We created these objects ourselves within actual cultural practices. These practices quite literally embody them - no further realm must be assumed. Abstracta must be learned, not found. They are also embedded within a causal nexus, for they have their roots in entanglements. Hence, they are objective in the sense of having an anchor in the world. They are causally connected to it twice: through a primary interaction which gave birth to this practice in the beginning and through a long history of passing on and refinement to later generations.

This proposal will not end debates about (anti-)Platonism, but it broadens the view. Otherwise unasked questions arise: first and foremost, how are abstract objects concretely materialized; how do they get instantiated in a way that allows them to embody their specific "abstract" features; and how do they both affect and function within the cognitive capacities of later hominins?

Furthermore, we do not believe in logical truths by default; even less so because we engage in deductive inferences by default when doing some reasoning. We do not, as a considerable amount of research has indicated in the last 30 years. However, we can be quickly educated in a way as to believe them quickly (Mody and Carey 2016). But without being exposed to learning logic in a cultural setting, no beliefs in logical objects occur. This indicates that "reliability" between beliefs and "facts" could be post hoc: without learning these abstracta, we do not know them; after learning them, we believe in their nature as being taught to us. If this is on the right track, a match is no miracle.

Finally, material artifacts with abstract content are not representations of otherwise unworldly abstracta. The latter are nothing but these are actual material items. A mathematical notation on a clay tablet shall not represent mathematical objects; it shall engage us in specific practices. ${ }^{18}$ There is no direct reference of logical terms to their mind-independent logical "objects." The laws of logic do not exist in themselves and can then be put into use by smart animals. Instead, any terms of logical systems we use are build up by use of our (particular) logical cognition, where this ability refers indirectly to a (particular) history of patterns of interaction between organisms, their specific needs, and their always changing niches.

This also renders the epistemological question idle: It is similar to asking for the epistemology of the rules of chess - or of ancient myths' characters. The question is not how we reliably know abstract entities; the question is how these entities, once created by intellectual craftsmanship, do play an essential role in gaining knowledge (and have played during the history of our cognitive abilities).

\footnotetext{
${ }^{18}$ See Menary (2015) for a full-blown account.
} 
Norms. A standard Fregean conception of logic as normative depends on a twin idea. First, norms for thought must be independent of any subject. Second, logic is normative for reasoning in general. Thus, abstract logical objects must be some general, non-contingent superstructure of our universe. I have argued that they are not. Granted deductive logic would have lost its metaphysically stable status, solicitudes would be manageable for our species, however: as argued above, within realworld scenarios, there is no need for the hominin mind to follow the rules of deductive logic, for it occurred no general need to follow "no counterexamples"-rules in their history. That such rules could rise as an intersubjective standard for a group embedded in specific environments and furnished with specific needs is rather unlikely-even if the members of this group are capable of domain-general cognition. They are not the one solely proper set of rational general reasoning norms normal hominins should aspire to in every situation. If so, they are not norms for reasoning in general. Instead, they are historically contingent artifacts, which have been designed for specific purposes. Even they depend on context: whether they are norms worth following depends on where one is going to use them, and whether it will work out at this specific place. ${ }^{19}$

\section{Concluding Remarks: Animal Needs and Hominin Niches}

Hominins are animals with needs. As I argued, this, as well as being embedded in a certain way of life - a niche - constitutes their cognitive makeup. Changing niches foster appearances of new cognitive abilities. In the case of the hominins, a new way of life of coordinated group activities gave rise to human logical cognition. Yet it did not - and could not - give rise to reason by deductive logic. Logical cognition thus must mean something different for our species than the latter.

So far, these remarks are sketchy. The challenge is open, however, to present an empirically grounded theory that links planning capabilities with deductive reasoning rules and not just a mere logical possibility that it could be so in some corner of the set of possible worlds. A lot is possible in that realm - and hence, it is of little to no help for finding out what had happened in our world during hominin cognitive evolution.

Already in this world, several phenomena are left to be explained: How specific abstracta evolved during hominin history-myths, scripts, mathematics, and so on - and how their features can be described in purely naturalistic terms. Also, how and why hominin history took its turns for these things, for it is one thing to explain how mythical, abstract, and likewise logical cognition became a requirement for the inhabitants of a specific environment. It is another part of the story to explain how the bearers of that kind of cognition ended up in that very environment in the first place.

So finally, there is still another worry. Even a plausible scenario is not enougheventually we must aim for a plausible story, i.e., an evolutionary trajectory: a series of small steps that reveal how animals with baseline capacities of the trait in question could turn into full-blown owners of it. For this, one would have to provide an

\footnotetext{
${ }_{19}$ This leads to an interesting point: If norms are to be considered context-sensitive due to our evolved nature, how is this to be reflected methodologically in debates on the normativity of logic? Due to lack of space, my stance on these issues will be the theme of a separate paper. (Thanks to an anonymous referee for pointing out the potential of this.)
} 
application of niche construction theory with the archeological evidence in stock to make this story complete. But this must be the theme of yet another paper. ${ }^{20}$ In any event, as demonstrated here, there is a plausible new perspective on the phenomenon. If true, it is a more plausible track to follow.

The moral here is not just a lesson in the epistemology of logic, however, for the main line of thought can be generalized to any human cognitive activity. Two purposes unfold as a future outlook: first, to delve more thoroughly into the role of material culture in Homo sapiens cognitive evolution and second, to delve more thoroughly into the role of abstract objects within these cognitive practices, that is, to review abstracta as artifacts.

Funding Information Open access funding provided by Projekt DEAL.

Open Access This article is licensed under a Creative Commons Attribution 4.0 International License, which permits use, sharing, adaptation, distribution and reproduction in any medium or format, as long as you give appropriate credit to the original author(s) and the source, provide a link to the Creative Commons licence, and indicate if changes were made. The images or other third party material in this article are included in the article's Creative Commons licence, unless indicated otherwise in a credit line to the material. If material is not included in the article's Creative Commons licence and your intended use is not permitted by statutory regulation or exceeds the permitted use, you will need to obtain permission directly from the copyright holder. To view a copy of this licence, visit http://creativecommons.org/licenses/by/4.0/.

\section{References}

Boivin, N. (2008). Material cultures, material minds. Cambridge University Press.

Bunn, H. T. (1994). Early Pleistocene hominid foraging strategies along the ancestral Omo River at Koobi Fora, Kenya. Journal of Human Evolution, 27(1), 247-266.

Bunn, H. T. (2007). "Meat made us human." Evolution of the human diet: the known, the unknown, and the unknowable (pp. 191-211). Oxford: Oxford University Press.

Bunn, H. T., \& Pickering, T. R. (2010a). Bovid mortality profiles in Paleoecological context falsify hypotheses of endurance running-hunting and passive scavenging by Early Pleistocene hominins. Quaternary Research, 74(3), 395-404.

Bunn, H. T., \& Pickering, T. R. (2010b). Methodological recommendations for ungulate mortality analyses in paleoanthropology. Quaternary Research, 74(3), 388-394.

Clark, J. D., \& Harris, J. W. K.. 1985. "Fire and its roles in early hominid lifeways." The African Archaeological Review 3 (1). Kluwer Academic Publishers: 3-27. https://doi.org/10.1007/BF01117453.

Coolidge, F. L., \& Wynn, T. (2012). How to think like a Neanderthal. Oxford University Press.

Cooper, W. S. (2001). The evolution of reason: logic as a branch of biology. Cambridge: Cambridge University Press.

Currie, Adrian, and Kim Sterelny. 2017. "In defence of story-telling." Studies in History and Philosophy of Science Part A 62: 14-21. doi:https://doi.org/10.1016/j.shpsa.2017.03.003.

Day, R. L., Laland, K. N., \& Odling-Smee, F. J. (2003). Rethinking adaptation: the Niche-Construction perspective. Perspectives in Biology and Medicine, 46(1). The Johns Hopkins University Press), 80-95.

Dutilh Novaes, C. (2012). Formal languages in logic. Cambridge University Press.

Dutilh Novaes, C. (2015). A dialogical, multi-agent account of the normativity of logic. Dialectica, 69(4), 587-609. https://doi.org/10.1111/1746-8361.12118.

Foley, R., \& Gamble, C. (2009). The ecology of social transitions in human evolution. Philosophical Transactions of the Royal Society B: Biological Sciences, 364(1533), 3267-3279.

Gallagher, S. (2017). Enactivist interventions. Oxford University Press.

Godfrey-Smith, P. (1996). Complexity and the function of mind in nature. Cambridge University Press.

\footnotetext{
${ }^{20}$ See Seitz (forthcoming).
} 
Godfrey-Smith, P. (2002). Environmental complexity and the evolution of cognition. In R. Sternberg \& J. Kaufman (Eds.), The evolution of intelligence (pp. 233-249). Mahwah: Erlbaum.

Godfrey-Smith, P. (2017). Complexity revisited. Biology \& Philosophy, 32(3), 467-479.

Goren-Inbar, N., Alperson, N., Kislev, M. E., Simchoni, O., Melamed, Y., Ben-Nun, A., \& Werker, E. (2004). Evidence of hominin control of fire at Gesher Benot Yaaqov, Israel. Science, 304(5671), 725-727.

Haidle, M. (2009). How to think a simple spear. In T. Wynn, F. L. Coolidge, \& S. A. de Beaune (Eds.), Cognitive archeology and human evolution (pp. 57-74).

Hay, R. L. R. (1976). Geology of the Olduvai Gorge: a study of sedimentation in a Semiarid Basin. University of California Press.

Henrich, J. (2016). The secret of our success. Princeton University Press.

Heyes, C. (2012). New thinking: the evolution of human cognition. Philosophical Transactions of the Royal Society B: Biological Sciences, 367(1599), 2091-2096. https://doi.org/10.1098/rstb.2012.0111.

Hodder, I. (2012). Entangled. John Wiley \& Sons.

Hodder, I. (2014). "The entanglements of humans and things: a long-term view." New Literary History 45 (1). The Johns Hopkins University Press: 19-36. https://doi.org/10.1353/nlh.2014.0005.

Hutchins, E. (1999). Cognitive artifacts. In R. A. Wilson \& F. C. Keil (Eds.), The MIT encyclopedia of the cognitive sciences. MIT Press.

Hutto, D. D., \& Myin, E. (2013). Radicalizing enactivism. MIT Press.

Jeffares, B. (2010a). The evolution of technical competence: strategic and economic thinking. In W. Christensen, E. Schier, \& J. Sutton (Eds.), Proceedings of the conference of the Australasian Society for Cognitive Science (pp. 162-169). Sydney: Macquarie Centre for Cognitive Science. https://doi. org/10.5096/ASCS200925.

Jeffares, B. (2010b). The co-evolution of tools and minds: cognition and material culture in the hominin lineage. Phenomenology and the Cognitive Sciences, 9(4), 503-520. https://doi.org/10.1007/s11097-0109176-9.

Laland, K. N. (2017). Darwin's unfinished symphony. Princeton University Press.

Laland, K. N., \& Brown G. R. (2006). Niche Construction, Human Behavior, and the Adaptive-Lag Hypothesis. Evolutionary Anthropology: Issues, News, and Reviews, 15(3), 95-104.

Laland, K. N., Odling-Smee, J., \& Feldman, M. W. (2000). Niche construction, biological evolution, and cultural change. Behavioral and Brain Sciences, 23(1). Cambridge University Press), 131-146. https://doi.org/10.1017/S0140525X00002417.

Leroi-Gourhan, A. (1965). Le Geste Et La Parole. Paris: Albin Michel.

Lloyd, G. E. R. (1996). Adversaries and authorities. Cambridge University Press.

Menary, R. (2007). Cognitive integration. Palgrave Macmillian.

Menary, R. (2015). Mathematical cognition: a case of enculturation. In T. Metzinger \& J. M. Windt (Eds.), Open MIND (pp. 1-20). Mainz: Johannes Gutenberg Universität.

Mercier, H., \& Sperber, D. (2011). Why do humans reason? Arguments for an argumentative theory. The Behavioral and Brain Sciences, 34(2), 57-74; discussion74-discussion111. https://doi.org/10.1017 /S0140525X10000968.

Mody, S., \& Carey, S. (2016). The emergence of reasoning by the disjunctive syllogism in early childhood. Cognition, 154(September), 40-48. https://doi.org/10.1016/j.cognition.2016.05.012.

Netz, R. (1999). The shaping of deduction in Greek mathematics. Cambridge University Press.

Ohel, M. (1984). Spatial management of hominid groups at Olduvai: a preliminary exercise. Paleoecology of Africa, 14, 125-146.

Osvath, M., \& Gärdenfors, P. (2004). Oldowan culture and the evolution of anticipatory cognition. Lund University Cognitive Studies, 122, 1-16.

Plummer, T. (2004). Flaked stones and old bones: biological and cultural evolution at the dawn of technology. American Journal of Physical Anthropologyl, 39(January), 118-164.

Potts, R. (1984). Home bases and early hominids. American Scientist, 72, 338-347.

Richardson, R. C. (2008). Evolutionary psychology as maladapted psychology. MIT Press.

Saslis-Lagoudakis, C. H., Hawkins, J. A., Greenhill, S. J., Pendry, C. A., Watson, M. F., Tuladhar-Douglas, W., Baral, S. R., \& Savolainen, V. (2014). The evolution of traditional knowledge: environment shapes medicinal plant use in Nepal. Proceedings of the Royal Society B: Biological Sciences, 281(1780). The Royal Society), 20132768-20132768. https://doi.org/10.1098/rspb.2013.2768.

Schechter, J. (2011). The reliability challenge and the epistemology of logic. Philosophical Perspectives, 24(1), 437-464. https://doi.org/10.1111/j.1520-8583.2010.00199.x Blackwell Publishing Inc.

Schechter, J. (2013). Could evolution explain our reliability about logic? Oxford studies in epistemology Volume, 4, 214-239. Oxford University Press. https://doi.org/10.1093/acprof:oso/9780199672707.003.0008. 
Seitz, F. (forthcoming). Argumentation evolved - but how?. Argumentation. https://doi.org/10.1007/s10503020-09510-6.

Semaw, S., Renne, P., Harris, J. W., Feibel, C. S., Bernor, R. L., Fesseha, N., \& Mowbray, K. (1997). 2.5million-year-old stone tools from Gona, Ethiopia. Nature, 385(6614), 333.

Smith, R. J. (2016). Explanations for Adaptations, just-so stories, and limitations on evidence in evolutionary biology. Evolutionary Anthropology, 25(6), 276-287. https://doi.org/10.1002/evan.21495.

Sober, E. (1981). The evolution of rationality. Synthese, 46(1), 95-120.

Stenning, K., \& van Lambalgen, M. (2008). Human reasoning and cognitive science. MIT Press.

Sterelny, K. (2007). Social intelligence, human intelligence and niche construction. Philosophical Transactions of the Royal Society of London. Series B, Biological Sciences, 362(1480), 719-730. https://doi.org/10.1098/rstb.2006.2006.

Sterelny, K. (2012). The evolved apprentice. MIT Press.

Suddendorf, T. (2013). The gap. Basic Books.

Sutton, J. (2010). Exograms and interdisciplinarity: history, the extended mind, and the civilizing process. In The extended mind (pp. 189-225). The MIT Press. https://doi.org/10.7551 /mitpress/9780262014038.003.0009.

Thieme, H. (1997). Lower Palaeolithic hunting spears from Germany. Nature, 385(6619), 807-810.

Tomasello, M. (2014). A natural history of human thinking. Harvard University Press. https://doi.org/10.2307 j.ctt6wpq11.

Villa, P., \& Lenoir, M. (2009). Hunting and hunting weapons of the Lower and Middle Paleolithic of Europe. In The evolution of hominin diets, Vertebrate paleobiology and paleoanthropology (pp. 59-85). Dordrecht: Springer Netherlands. https://doi.org/10.1007/978-1-4020-9699-0 5.

Walter, Sven. 2009. "Robert C. Richardson: Evolutionary psychology as maladapted psychology." Mind, Volume 118, Issue 470: 523-527.

Wrangham, R. W. (2010). Catching fire. Profile Books.

Wrangham, R., \& Conklin-Brittain, N. L. (2003). Cooking as a biological trait. Comparative Biochemistry and Physiology Part A: Molecular \& Integrative Physiology, 136(1), 35-46. https://doi.org/10.1016/S10956433(03)00020-5.

Publisher's Note Springer Nature remains neutral with regard to jurisdictional claims in published maps and institutional affiliations. 